\title{
Variations
}

Variations

Revue internationale de théorie critique

$13 / 14 \mid 2010$

Le choix du petit

\section{Rupture de la pédagogie et pédagogie de la rupture}

Frank Jablonka

\section{OpenEdition}

Journals

Édition électronique

URL : http://journals.openedition.org/variations/187

DOI : 10.4000/variations. 187

ISSN : 1968-3960

Éditeur

Les amis de Variations

Édition imprimée

Date de publication : 31 mars 2010

\section{Référence électronique}

Frank Jablonka, «Rupture de la pédagogie et pédagogie de la rupture », Variations [En ligne],

13/14 | 2010, mis en ligne le 01 février 2012, consulté le 03 mai 2019. URL : http://

journals.openedition.org/variations/187; DOI : 10.4000/variations.187

Ce document a été généré automatiquement le 3 mai 2019.

Les ami•e•s de Variations 


\title{
Rupture de la pédagogie et pédagogie de la rupture
}

\author{
Frank Jablonka
}

\section{NOTE DE L'ÉDITEUR}

Première publication sur www.theoriecritique.com, « Le choix du petit », Printemps 2010, pp. 141-159

\section{Discours et contre-discours de la rupture}

1 1.1. La présente contribution a pour but de proposer quelques considérations intempestives par rapport aux conceptions pédagogiques dominantes et à la politique éducative actuellement mise en œuvre en France. Si les problèmes ne sont, certes, pas nouveaux, ils ont été exacerbés depuis 2007, conjointement aux conceptions sous-jacentes et à leur mise en œuvre autoritaire et "décomplexée" par le nouveau gouvernement. Ce symptôme de décadence apparaît dans les discours sociaux conjointement à un terme qui connaît actuellement un usage quelque peu inflationnaire, à savoir de crise. Dans la mesure où l'ensemble social subit des tendances de désintégration, où l'on craint une érosion normative et une désorientation socioculturelle, les autorités en place cherchent à 'serrer les vices' en appliquant des mesures et directives rigoureuses, voire rigoristes (cf. Marcuse, 2008a, 90). C'est précisément contre cet air du temps que se dirigent nos réflexions ; c'est contre la mouvance hégémonique conforme à l'esprit de la bourgeoisie autochtone qu'il s'agit ex negativo de jeter les bases d'un projet éducatif contrehégémonique, précisément par la critique de l'ordre de valeurs établi et de l'ordre social en vigueur.

2 1.2. Depuis la même période, qui représente effectivement à maints égards une césure, on entend également, de manière récurrente, un discours de "rupture" (cf. Badiou, 2007, 103 
ss.). C'est, en effet, dans une mesure importante, sur le monde des valeurs que l'attention des dirigeants en place depuis 2007 est portée, c'est-à-dire sur l'ordre moral, situé au cœur des compétences pédagogiques. Si c'est sur les valeurs soixante-huitardes (Badiou, 2007, 108), ou plutôt sur ce qu'il en reste après une quarantaine d'années de dépolitisation systématique, que le Président souhaite "tourner la page", ce n'est certainement pas parce que la mise en place d'une contre-hégémonie culturelle, véhiculée par le système éducatif, serait imminente. Pour que le projet de société néolibéral puisse achever son œuvre en profondeur, il est nécessaire d'achever la dernière potentialité d'une opposition politique et d'une contestation idéologique fondamentales. "Sarkozy annonce qu'il va surmonter la crise morale de la France, il va la remettre au travail" (Badiou, 2007, 105): asseoir l'hégémonie culturelle néolibérale, corollaire indispensable de l'hégémonie politique incontestablement acquise, implique de rendre toute alternative d'un autre monde, altermondialiste, précisément, impensable. La "pensée positive", contre laquelle s'insurge Marcuse (1968), remporte sa victoire définitive lorsque sa positivité n'est plus perceptible, c'est-à-dire lorsque toute négativité sur l'arrière-plan de laquelle une critique de principe pourrait être formulée, sera effacée. L'objectif de la politique culturelle, notamment éducative en vigueur est de dérober la critique non seulement de tout ancrage institutionnel, mais surtout de ses repères conceptuels. L'enjeu est d'ordre biopolitique (Hardt/Negri, 2004) : ce qui est en jeu est précisément la (re)production de la vie elle-même, en l'occurrence de sujets dociles et fongibles. Si, dans cette situation, il faut en effet chercher longtemps avant d'apercevoir de vagues semblants d'issues; si la contingence de l'hégémonie plus fermement assise que jamais est loin de sauter aux yeux, c'est précisément en raison de l'absence de tout projet opérationnel de contre-hégémonie (Mouffe, 2008, 33). Même si nous ne croyons plus aujourd'hui, comme c'était encore le cas dans les années soixante-dix, au rôle de la pédagogie comme science pilote de la transformation émancipatoire de la société capitaliste, il apparaît néanmoins prometteur d'évaluer les potentiels pédagogiques pour la mise en place au moins de l'amorce d'un tel projet. Il s'agit maintenant de se donner les moyens de réhabiliter le terrain des institutions et établissements éducatifs comme champ de la lutte de classe sur le front idéologique.

3 1.3. Pour ce faire, le premier pas est sans doute la récupération du terme de "rupture" même, usurpé par le discours dominant. Dans ce but, il s'agira de désarticuler ce dernier. Si la logique de la rupture se présente sous la forme politique de (contre-)réformes, il est nécessaire de rompre avec ces ruptures néolibérales. Le type de rupture auquel nous faisons allusion est en l'occurrence celui de clinamen; c'est précisément dans cette acception que le terme de "rupture" dans le titre est à interpréter : la rupture de la pédagogie et la pédagogie de rupture s'entendent comme clinamen au sens de changement du cours des choses "normal" par une action délibérée et non prévisible (d'où son caractère "intempestif"), qui aboutit à l'émergence de phénomènes et d'événements d'un type nouveau: "the moment of rupture or clinamen that can create a new world. [...] The bowstring shoots the arrow of a new temporality, inaugurating a new future." (Hardt/ Negri, 2004, 357) C'est ainsi dans la discontinuité du temps historique, de la rupture entre le temps continu (chronos), ce long fleuve tranquille peuplé de crocodiles, et la foudre momentanée du kaïròs, où la classe dominée est amenée à chercher son opportunité pour l'éclat d'une lutte victorieuse, discontinuité qui permet de sortir d'une évolution intrinsèquement catastrophique. Sans vouloir évoquer la vision mythologique, sinon messianique de la grève générale, dont Georges Sorel (1990) est l'auteur, il est tout à fait imaginable que la lutte s'articule concrètement sous cette forme, y compris, et de façon 
privilégiée, dans le secteur public, terrain principal de l'action pédagogique et de la mise en œuvre de la politique éducative. À titre d'inspiration de l'entrée dans une logique contestataire d'ampleur, nous recommandons, avec Walter Benjamin (2007, 321), le "saut de tigre dans le passé" ("Tigersprung"), qui donne en même temps l'ouverture projective et anticipatrice sur l'avenir. Si nous précisons que pour Benjamin, ce saut de tigre est le saut dialectique de la révolution au sens marxien, même si pour l'instant le théâtre en est toujours, comme le reconnaît Benjamin lui-même, le terrain contrôlé par la classe dominante, il va sans dire que ce passé proche de la dernière grève générale d'envergure quasi-révolutionnaire est le même mai 68 sur lequel le discours présidentiel souhaite tourner la page. Il est nécessaire de revivifier ce passé qui nous donne une nouvelle page, sinon un chapitre d'histoire à venir, à écrire.

1.4. Comme le discours de "clinamen" et de "kaïros" le suggère, ce "saut de tigre" à besoin d'un support motivationnel mythique. En effet, Maffesoli $(2007,14)$ souligne à juste titre que "la rébellion latente ou explosive, les pratiques à risques" ne sont pas - ou en tout cas pas exclusivement, "les manifestations d'une simple misère sociale", même lorsque celleci en est le déclencheur manifeste. Un ressourcement transgressif d'ampleur représente nécessairement aussi, sur le plan symbolique, une rupture archétypique correspondant à une coupure libératrice qui ouvre de nouveaux horizons de sens et de pratique et qui permet de localiser l'utopie dans le hic et nunc. C'est précisément sur cette charge mythologique indispensable que repose la revendication tout à fait réaliste de l'impossible, évoquée par la commémoration soixante-huitarde.

5 1.5. Dans ceci, une erreur à éviter à tout prix serait l'imitation de la stratégie militaire américaine au Vietnam, qui consistait, soi-disant, dans la destruction de villages habités pour en libérer la population. Il y a une dizaine d'années déjà, Pierre Bourdieu $(2001,38)$ a esquissé le paradoxe devant lequel l'action syndicale est placée face à la politique éducative néolibérale : il faut défendre l'école publique par tous les moyens, même si elle mérite la critique la plus impitoyable, du fait de son caractère conservateur et de sa fonction stabilisatrice du système de société existant. Face à ce dilemme, nous ne pouvons pas nous empêcher de souligner l'évidence que la préservation des services publics, dont le secteur éducatif dans son ensemble, est effectivement empêtrée dans un cul-de-sac, à moins que l'on en envisage du même coup la transformation fondamentale dans la visée émancipatrice de démocratisation en profondeur. Il va de soi que ce secteur, dont le cœur est traversé par le front idéologique, est déjà, et le sera encore davantage dans un proche avenir, le terrain privilégié de la politique d'émancipation.

\section{Action pédagogique et action politique au sein des "Appareils Idéologiques d'État"}

6 2.1. Dans ce cadre est investi tout le champ des "Appareils Idéologiques d'État" (AIE, cf. Althusser, 1976), c'est-à-dire des appareils dont la fonction est de reproduire les conditions de production au sein de l'économie du marché capitaliste, base matérielle du fonctionnement de l'État bourgeois. Ceci implique que la conscience des sujets soit assujettie, précisément, aux impératifs de fongibilité de ce mécanisme. Tout le système éducatif, de l'école maternelle à l'école postdoctorale, est intégralement investie dans ce processus de reproduction idéologique - ce qui explique la particulière sensibilité du champ pédagogique aux yeux des appareils répressifs, bien qu'il ne soit pas productif en lui-même. La fatalité est que les instances relevant du champ idéologique déploient et 
suivent leur dynamique propre, ce qui permet une certaine indépendance vis-à-vis des autorités de contrôle. D'autre part, ces marges d'incontrôlabilité exacerbent la méfiance des instances qui veillent jalousement sur la préservation de l'hégémonie culturelle bourgeoise. Cette sensibilité est une chance, car elle permet de déjouer les exigences d'immobilité de l'appareil et de retourner la dynamique de fonctionnement contre celuici, tout en bénéficiant - dans de très nombreuses situations - toujours de la protection garantie par le statut de fonctionnaire. Il faut, dans ce contexte, rappeler le fonctionnement intrinsèquement contradictoire des AIE, grâce auquel s'ouvrent de nombreuses failles qui s'offrent à l'action contestataire: en vertu de leur fonction de reproduction des bases idéologiques des conditions de production, les AIE sont en même temps aussi immanquablement la condition de la transformation de celles-ci (cf. Pêcheux, $1984 a, b)$, et un terrain propice de surcroît. Pêcheux $(1975,131)$ cite comme exemple paradigmatique les institutions scolaires placées entre les intérêts parentaux et les exigences de l'économie du marché, donc généralement en interaction avec d'autres AIE, qu'il faut, en réalité, voir dans leur synergie complexe. Les AIE étant des lieux contradictoires de la reproduction de l'idéologie dominante, qui ne fonctionne jamais par " copié-collé ", ils portent en eux-mêmes les conditions de leur requalification en terrains de politique contestataire. C'est au sein des AIE, avec le système éducatif en première file, qu'a lieu la lutte pour l'imposition de nouvelles relations de domination - et de subordination - et donc aussi d'insubordination. Ainsi, le caractère intrinsèquement agonistique de l'interaction sur le champ politique, et la dynamique transformatrice, peut être clairement (mais certainement pas exclusivement) situé au sein des AIE: "Adversaries inscribe their confrontations within the democratic framework, but this framework is not seen as something immutable: it is susceptible of being redefined through hegemonic struggle.» (Mouffe 2008, 32 s.) Une partie importante de ces luttes se joue dans le domaine de l'inconscient par processus symboliques interposés. Quant au système scolaire, il apparaît, en effet, que la gravure des programmes de l'idéologie dominante dans les couches inconscientes est considérablement profonde et durable mais peut-être pas pour autant complètement indélébile.

7 2.2. Le mécanisme socio-psychologique, qui représente la clé du fonctionnement des AIE, est l'introjection de la structure normative et de l'idéologie dominantes en vigueur dans la structure mentale et inconsciente des sujets et la soumission des sujets à celles-ci. En vertu des AIE, nous portons le système, nous portons l'État en nous. Pour que ce mécanisme puisse fonctionner, il exige, comme objectif pédagogique prioritaire, l'identification avec le système, avec l'État. Pour être plus précis, la constitution des sujets (en l'occurrence " citoyens », fonctionnaires ou non) en tant que tels est une fonction des AIE : ils sont modelés à leur image. Cependant, ce formatage n'est pas exempt d'un certain taux de ratage heureux. Ce phénomène collatéral, mais inévitable, est source permanente d'instabilité au sein du système, et ainsi une précieuse ressource de contestation à mobiliser de l'intérieur. L'internalisation des normes et valeurs dominantes, inévitablement vécue comme processus répressif, ne peut être rationalisée sous l'étiquette idéologique d' " éducation » que dans la mesure où les sujets sont soumis, et se soumettent eux-mêmes, à une opération d'«identification avec l'agresseur " (cf. Osterkamp, 1999, 1333). Par ricochet, et sous forme du "retour du refoulé», cette agression se dirige contre ceux qui, à l'intérieur du système même, apparaissent comme inadaptés, ou moins adaptés, ce qui revient, dans un contexte général de "réforme " néolibérale de la fonction publique, dans de nombreux cas à la remise en question de leur 
aptitude. En contrepartie, le rappel vivant de la contingence de l'hégémonie que ces « ratés » incarnent est susceptible de remettre en question l'adaptation de la majorité et d'éveiller les douleurs qui ont fait d'eux ce qu'ils sont. Etant donné que le processus d'introjection repose sur le rejet de potentiels de développement et d'épanouissement personnels, conformément aux impératifs restrictifs du système, c'est le reflet de leur propre vie non vécue que les adaptés, et notamment leurs responsables, aperçoivent dans le miroir de la contingence. La stratégie consiste ainsi à capitaliser le potentiel subversif de la commémoration vivante que représente l'Autre en qualité de contingence incarnée. C'est précisément cette altérité que les bras longs des AIE - des fonctionnaires suffisamment haut placés pour participer du pouvoir de l'élite dominante, sans pour autant avoir accès aux mécanismes de régulation des principes de fonctionnement, normes et valeurs auxquelles l'appareil est soumis: chefs d'établissements, inspecteurs académiques, fonctionnaires du rectorat, etc. - cherchent à étouffer par tous les moyens, en restreignant les marges de manœuvre participatives des porteurs de cette altérité. A contrario, nous identifions dans cette catégorie de personnel, du fait de sa frustration structurelle liée à sa position intermédiaire et par conséquent ambivalente - et de surcroît en situation de crise en stade avancée - une faille dans le système propice à attirer, comme un aimant, tous les potentiels contestataires sur elle. Cette faille est susceptible de s'ouvrir dans la même mesure où l'insatisfaction à tous niveaux de l'appareil devrait s'agrandir dans les prochaines années sous l'effet du "dégraissage » 'éléphantesque' et progressif des différents corps de fonctionnaires annoncé par les autorités gouvernementales, sans parler des agents non titulaires. Il est entendu que cette politique touche déjà, et touchera dans l'avenir encore davantage, l'infrastructure institutionnelle couvrant les couches populaires, déjà fragilisées.

8 2.3. Pour inverser la relation de probabilité, qui joue à l'état actuel toujours en faveur de l'adaptation et de l'installation dans l'hétéronomie, et non pas de la contestation, la stratégie recommandée est par conséquent de mettre en mots ce complexe, apparemment privé, de désirs, émotions, espoirs, mais aussi de déceptions, de frustrations et de désespoir, et d'en faire apparaître le caractère foncièrement social - et ceci en situation et au sein des AIE, au cœur des institutions éducatives et des processus pédagogiques (cf. aussi Adorno, 1971, 83). Il est cependant probable que ce serait, au moins dans un premier temps, une avalanche de malaise qui ressort, point de départ de la mobilisation de résistances, voir de la Résistance - même s'il faut s'attendre aussi à d'autres types de résistances, assez importantes, y compris à la perception: étant donné que la verbalisation d'émotions « critiques » est, comme l'affirme Osterkamp (1999, 1333 s.) à juste titre, un « acte révolutionnaire » dans une société de classes, de telles pratiques se heurteront forcément à des manœuvres de déréalisation, voir d'irrationalisation des contenus affectifs respectifs.

9 2.4. Car il ne faut surtout à aucun moment oublier l'importance et le caractère des obstacles psychosociaux qui s'opposent à cette tâche nécessaire. Si l'autorité hégémoniale est fermement et profondément ancrée dans l'appareil psychique, c'est en grande partie l'œuvre des dispositifs disciplinaires des AIE visant la subordination des sujets comme automatisme au sens d'internalisation "d'habitudes, de pratiques, de besoins et des relatives formes de satisfactions selon les modes de vie moyens des hommes » (cf. Haug, 1999, 789 ss.). La conséquence est une restriction de l'autonomie d'action, correspondant à un figement dans une fongibilité préformée aliénée, sans que ce syndrome soit pour autant nécessairement accessible à la conscience des sujets, car le mécanisme fait 
efficacement barrage aux éventuelles velléités de pensée critique substantielle. C'est sans doute en cette inculcation que consiste le « programme occulte » qui s'inscrit, sans jamais avoir été écrit, dans le système éducatif et scolaire public, imposé par voie de procédures pédagogiques ritualisées (Illich, 2003, 63 et passim). Suivant les conclusions psychopédagogiques de l'école critique berlinoise, tout à fait applicables au cas de figure français, l'éducation scolaire freine structurellement l'apprentissage « expansif » (visant l'autonomie d'action, conjointement à l'ouverture cognitive sur le monde de la part des apprenants) et est, de ce fait, soumise à une "dégradation de la culture éducative » (Auernheimer 1998, 838).

10 2.5. Ce qui complique considérablement la tâche à une pédagogie émancipatrice de rupture, c'est que l'émancipation nécessite à son tour une posture disciplinée (cf. Haug, 1999, 789); pire encore, celle-ci est d'autant plus indispensable - et par ailleurs défaillante - que l'opposition à l'hégémonie culturelle, qui anime les AIE et tout le tissu institutionnel public qui en dépend, nécessite décidément une fermeté rigoureuse. On peut se faire une idée des dimensions de cette tâche si on prend en considération la réflexion de Friedrich Engels (MEW 18, 307) selon laquelle une opération plus autoritaire qu'une révolution n'est guère imaginable. Sur cet arrière-plan, le dilemme qui s'oppose à la pédagogie, même émancipatrice, et qui en exige en conséquence la rupture, apparaît en toute clarté: les élèves, respectivement étudiants, assimilent les tentatives de disciplinarisation libératrice aux dispositifs de formatage disciplinaire autoritariste administré selon la logique de l'appareil dominant. Au lieu de rejeter cette logique répressive mise en œuvre par les agents au service de l'hégémonie, ils rejettent du même coup toute velléité contre-hégémonique. Ce problème est sans doute aussi lié à d'anciens stéréotypes qui s'attachent au rôle de l'enseignant, quasiment indéracinables dans la société bourgeoise (Adorno, 1971, 78 ss.). W. Reich (1974) caractérise précisément le double bind de la soumission à l'autorité simultanément reconnue et contestée comme caractéristique de la structure petite-bourgeoise qui représente en même temps l'humus de la culture politique autoritariste, voire de dérapages totalitaires, aucunement étrangers à l'histoire du gaullisme. La quête d'identifications-refuges avec des collectifs importants est un corollaire de ce syndrome (Adorno, 1971, 17). Sous cet angle, il est aisé d'assigner le rôle aux insécurités patriotiques et aux autres troubles psycho-affectifs du même registre, qui conduisent droit vers la création d'un ministère complètent de ce syndrome, dans l'ensemble de cette logique sous-jacente. Nous identifions dans ce mécanisme la clé qui nous permet de comprendre la nature de la rupture politique et culturelle intervenue en 2007, qui laisse, en effet, apparaître le pétainisme comme le «transcendantal», selon le diagnostic perspicace de Badiou (2007, 103 s.): «quelque chose qui, sans apparaître à la surface, [...] configure de loin la loi et son ordre, à une disposition collective. »

11 L'hégémonie s'installe ainsi dans une situation gagnant-gagnant. Et la pédagogie émancipatrice, quant à elle, est fatalement condamnée à un mouvement doublement à contre-courant: à la pression légitimatrice exercée tant par l'appareil (personnel d'encadrement, hiérarchie administrative, dans de nombreux cas aussi les collègues de l'équipe pédagogique) que par les étudiants respectivement élèves (et leurs parents, dans le secondaire). C'est ce que j'appelle le 'pentagone de la perdition'.

12 2.6. Dans ce pentagone, la famille est sans aucun doute l'AIE le plus percutant; conjointement avec le système éducatif public, elle assure en synergie le rôle de la reproduction de la structure sociale capitaliste et, du même coup, du capital. Le freudo- 
marxisme (cf. Lichtman, 1999, 1012 s.), depuis les écrits de Wilhelm Reich (1974) en passant par l'École de Francfort (Marcuse, 1963 et 1968 ; Adorno 1971 et 2007), insiste sur le moulage caractériel des enfants conformément aux exigences de la structure socioéconomique, en modelant la disposition des pulsions en conséquence, notamment dans un sens d'hétéronomie vis-à-vis des autorités, les préformant ainsi à leur future subordination dans le rôle de salariés. Le bras de fer avec le système économique, avec ses exigences de qualifications professionnels et dispositions psychologiques et comportementaux « utilisables ", qui prend les jeunes en tenaille, s'impose avec toute sa violence. Ceci est particulièrement manifeste dans le cas de la famille petite-bourgeoise qui, d'après Roland Barthes (1981, 167), détient en France (et sans doute aussi dans d'autres pays) l'hégémonie culturelle. À l'instar de la Matrix, machine de reproduction systématique de fausse conscience, alimentée malgré eux par la propre énergie des sujets, la famille correspond ainsi à la " main du créateur » de l'État capitaliste dans le système psychique et cognitif de la jeunesse : « if they were to refuse the relationship of power, to subtract themselves from the relationship, it would simply collapse in a lifeless heap. The film trilogy The Matrix interprets this dependence of power. The Matrix survives not only by sucking the energy from millions of incubated humans but also by responding to the creative attacks of Neo, Morpeus, and the partisans of Zion. The Matrix needs us to survive. » (Hardt/Negri, 2004, 335)

2.7. L'analyse de certains matériels pédagogiques permet de faire ressortir avec une clarté extraordinaire le caractère mystificateur qui repose sur la collaboration ritualisée des sujets soumis à l'éducation. Comme le montre Haug (1999, 789 s.), la disciplinarisation repose sur les pratiques standardisées d'entraînement à des représentations conformes aux exigences de fonctionnement de l'appareil et du système dans son ensemble, notamment dans l'interaction avec des appareils familiaux, économiques et administratifs. Un cas tiré d'un manuel d'Allemand (Decocqman et al. 2007, 41), très utilisé en classe de première, permet d'expliciter ce mécanisme idéologique de manière exemplaire. Il s'agit d'un exercice grammatical de manipulation formelle (c'est moi qui traduis, F.J.) :

Ex. 5 : Complétez les phrases à l'aide d'un relatif. Traduisez, après correction, le résultat obtenu.

Pinneberg est le meilleur vendeur ... ils ont chez Mandel, le magasin de mode. Il remarque tout de suite les gens ... ont assez d'argent pour s'acheter un nouveau pantalon ou une nouvelle veste. Par contre, son collègue Lasch, ... tout le monde appelle « le pauvre Lasch », est beaucoup trop timide. Il n'ose pas interpeller les clients ... regardent les vêtements. Qu'est-ce qui lui arrivera quand la nouvelle méthode, ... tout le monde parle en ce moment, sera appliquée ? Car un vendeur ... ne réussit pas à vendre des marchandises de $400 \mathrm{DM}$ par jour minimum sera licencié.

Ensuite, à la même page, le manuel propose une activité du même registre didactique et idéologique (traduction de l'auteur, F.J.) :

Ex. 6 : As-tu bien vendu?

Associez à l'aide de wenn [si] des éléments de la première série à des éléments de la deuxième série pour obtenir un résultat cohérent. 
Ex. : Si un vendeur ne vend pas telle ou telle quantité de marchandises, il sera licencié.

A. un vendeur / ne pas vendre telle ou telle quantité de marchandises

B. l'entreprise / ne pas faire de bonnes affaires

C. les salariés / ne pas réagir

D. les prix / continuer à monter

E. Pinneberg / vouloir rester chez Mandel

a. souvent ne plus avoir de droits

b. devoir vendre plus que ses collègues

c. un pain / coûter bientôt un million de DM

d. devoir fermer

e. être licencié

2.7.1. Ce qui ressort de cet extrait de matériel pédagogique au niveau idéologique, qui parle d'ailleurs pour lui-même, est la préparation à une future activité commerciale subalterne (en l'occurrence dans la vente), conjointement à la valorisation de celle-ci, comme s'il n'existait rien de plus naturel (la naturalisation idéologique de faits sociaux), notamment sur le plan de l'esprit capitaliste («l'entreprise / ne pas faire de bonnes affaires »); l'idéalisation de la logique productiviste («Si un vendeur ne vend pas telle ou telle quantité de marchandises, il sera licencié. ») ; l'utilisation de la peur pour l'emploi et la pression psychologique qui s'ensuit («Pinneberg / vouloir rester chez Mandel»); le culte de la concurrence ("devoir vendre plus que ses collègues»); le fétichisme de la monnaie forte par l'évocation du spectre inflationniste des années vingt, utilisé comme moyen de pression sur les salariés et comme justification de la modération salariale (« un pain / coûter bientôt un million de DM »); la (contre-)réforme néolibérale du droit du travail (« souvent ne plus avoir de droits ») ; réaction des salariés attendue en termes de productivité, certainement pas de militantisme syndical (« les salariés / ne pas réagir »).

2.7.2. Cette analyse n'est certainement pas exhaustive. Si c'était un manuel d'anglais, on commenterait sans hésiter : «Welcome to the Machine. » Mais même en allemand, la voix de la «Matrix» est bien audible. Comme le souligne Illich (2003, 90 s.) : "Tant que l'individu ne possède pas une conscience claire du caractère rituel du système par lequel il fut initié aux forces qui modèlent son univers, il est incapable de briser l'enchantement et de définir un nouveau 'cosmos'. Tant que nous ne prendrons pas conscience du rite par lequel l'école forme l'homme condamné à la consommation du progrès, il nous est impossible de briser le cercle magique et de faire apparaître une économie nouvelle. » C'est précisément cette prise de conscience qui est l'objectif de la présente analyse. Voyons alors ce qui se passe si nous refusons notre collaboration à la « Machine "; si nous sortons de la Matrix et si nous entrons enfin dans une logique de rupture et dans une pédagogie qui y correspond, afin de récupérer notre autonomie d'action en tant qu'enseignants et de la transmettre aux publics qui nous sont confiés. 


\section{Activité pédagogique comme engagement politique (et vice versa)}

17 3.1. Si l'objectif de l'action pédagogique au sein des AIE est la soumission, le premier objectif de la pédagogie de rupture doit être, a contrario, l'insoumission, afin de récupérer l'autonomie d'action. Si le «saut de tigre » nous fait atterrir à l'époque (post-)soixantehuitarde, nous nous ressourçons dans une conception éducative qui a recherché non pas l'adaptation docile aux exigences systémiques, mais qui a revendiqué le refus programmatique de se plier aux impératifs des autorités : « éducation à la désobéissance » (Bott, 1970). En revanche, les objectifs principaux de cette pédagogie, qui renonce à toute pratique contraignante, sont des compétences qui s'articulent en termes de critique, résistance, solidarité et empathie, à l'opposé de l'esprit concurrentiel, loin d'une préparation au productivisme qui attend dans la vie professionnelle capitaliste (ibid., 87, $90,100)$. Cette pédagogie mise sur le principe de l'autorégulation. Plus les marges de manœuvre sont larges et élastiques, plus les structures psycho-sociales émergentes sont riches. Il est cependant à souligner que ce principe présuppose l'anticipation d'une culture émancipée dans une société démocratique: seuls des individus habitués à la liberté pourront être porteurs d'une société libérée. On ne s'étonnera pas que lorsque les restrictions sont - soudain - levées, de nombreux enfants, notamment ceux qui sont soumis à un ordre contraignant dans leurs familles, réagissent dans un premier temps bruyamment par un comportement chaotique, difficilement compatible avec la phobie bourgeoise contre tout ce qui semble de près ou de loin quelque peu désordonné. Il faut, en revanche, insister sur le droit des enfants à une catharsis thérapeutique à laquelle succédera une phase pendant laquelle les enfants se donnent eux-mêmes des règles et commencent à réguler leurs besoins par eux-mêmes (principe de order from noise de l'auto-organisation systémique). Cette dynamique auto-éducative, qui efface l'éducateur et qui représente un entraînement à l'aplanissement hiérarchique, est difficile à accepter pour tous ceux à qui la nécessité d'un ordre autoritaire a été inculqué pendant leur propre éducation, qu'ils risquent par conséquent de reproduire dans leur propre pratique éducative. Ce type d'éducateurs, tant professionnels que parents, est soumis à des effets d'insécurisation profonde, voire à l'« humiliation de leur narcissisme » (Bott, 1970, 110).

3.2. Les enfants, eux, par contre, sûrs d'eux-mêmes, s'expriment librement et sans crainte. Si on ne leur impose pas l'obéissance, il n'y a aucun sens de les considérer comme désobéissants. Une telle attitude est la condition de la « dés-identification » sur laquelle insiste Pêcheux $(1975,215$; 1984, 63 ; cf. Barben, 1999, 1152), processus inverse du processus identificatoire avec l'ordre social, moral et étatique, objectif pédagogique discuté par rapport au processus psychosocial d'« introjection», œuvre des AIE. Pour compléter le panorama, cette stratégie poursuit en outre l'objectif de la sensibilisation aux rapports d'exploitation et d'inégalité socioéconomiques et socioculturelles dans la société capitaliste et prépare ainsi à des activités destinées à réaliser les présuppositions socio-pédagogiques anticipées. Il n'est, par conséquent, pas surprenant si certains parents s'inquiètent des conflits qui ne peuvent pas ne pas surgir, à moyen terme, avec l'environnement social, notamment scolaire, car l'anticipation nécessaire d'une culture émancipée dans une société démocratique est évidemment, pour l'instant, hélas, contrafactuelle. Ces craintes font partie des obstacles qu'une pédagogie de rupture, qui fonctionne doublement à contre-courant, doit inéluctablement prendre en compte. 
19 3.3. Il s'ensuit ainsi la nécessité d'une orientation à contre-courant permanent dans le quotidien, y compris contre soi-même, contre les idées reçues, contre le système introjecté, l'État que nous portons, nous tous, en nous, et, bien sûr, contre la tentation à la résignation, à la trahison de soi-même et des proches. Pour être crédibles et convaincants en tant que porteurs d'une pédagogie de rupture, il est nécessaire que la rupture soit consommée au sein des subjectivités. Une constance sans faille dans cette posture négative est indispensable pour "positiver» le rôle pédagogique dans une orientation contre-hégémonique : transformer le 'pentagone de la perdition' (cf. 2.5.) en 'pentagone de la libération' par le contact immédiat, y compris émotif, avec les subjectivités des autres.

20 C'est dans cette logique qu'est valable le mot d'ordre lancé par Pêcheux (1984a,b) face aux AIE : « oser se rebeller et penser ». Il s'agit là, de toute évidence, d'une adaptation du mot d'ordre émancipateur kantien "sapere aude», de l'injonction d'oser se servir de son propre intellect, adapté à la situation du capitalisme avancé (cf. pour la discussion aussi Marcuse, 2008, 36). Hardt/Negri $(2009,17)$ soulignent cependant que le corollaire de ce mot d'ordre kantien est une inversion qui souligne la nécessité de « savoir oser ». C'est en effet dans ce sens qu'il nous semble opportun de radicaliser cette perspective en reformulant ce mot d'ordre avec Barben $(1999,1153)$ une fois de plus : « oser savoir et se battre ». Ce serait dans ce sens-là que l'éducation à l'autonomie et à la critique préconisée par Adorno (1971) porterait le plus sûrement ses fruits.

21 3.4. Cette perspective implique la nécessité de rompre avec le néolibéralisme lui-même. Et si le mot d'ordre d'« affrontement social » a déjà été lancé, et non pas par l'opposition, mais par le gouvernement français, en personne du Président de la République lui-même, le caractère "agonistique » que Chantal Mouffe $(2008,33)$ attribue généralement au champ politique démocratique apparaît (enfin!) avec beaucoup plus de clarté que peu de temps avant. Dans la mesure où la tension monte, les camps adversaires se voient, eux aussi, poussés à agir, ce qui élargit considérablement, de part et d'autre, le champ des possibles. Le moment pour la gauche critique «to challenge power relations [...] through a process of disarticulation of existing practices and creations of new discourses and institutions » (Mouffe 2008, 33) semble ainsi atteindre des dimensions de plus en plus réelles et concrètes.

3.5. Si nous parlons d'une pédagogie de rupture en vue d'un projet contre-hégémonique, il faut entendre par là l'unité de politique - en l'occurrence anticapitaliste - et de pédagogie : comme le disait Marx lui-même dans sa troisième Thèse sur Feuerbach (MEW 3, 5-6), l'activité de changement des conditions de vie et celle du changement de soi-même, entendu comme constitution de soi-même comme sujet, sont coextensives dans la mesure où il s'agit de pratique révolutionnaire (pour la discussion cf. Birkmann/Winkler, 1998, 15), et impliquent ainsi une visée de révolution culturelle. Non seulement la politique est aussi une pratique éducative, le champ politique étant l'éducateur suprême, mais la pédagogie est aussi intégrée dans le projet d'une société libérée. Rappelons au passage qu'un tel « changement de soi » auto-éducatif n'a aucun rapport avec ce que l'on pourrait, dans la logique dominante, éventuellement entendre par "adaptation ", mais bien avec des modalités d'« être soi-même " et d' "être nous-mêmes " jusqu'à présent inédites et inexplorées. Cela implique l'interaction démocratique et émancipée, et surtout déhiérarchisée de la relation pédagogique, un vrai partenariat qui donne la parole à l'élève / à l'étudiant et qui impose à l'enseignant de descendre de son piédestal pour se mettre dans le rôle de l'apprenant. Dans cette pratique, ce sont l'État et sa classe 
dirigeante qui font l'objet d'une éducation, qui risque d'être, en l'occurrence, plutôt robuste. Voilà l'autre face, la vraie face, de l'Éducation Nationale au sens propre du terme (cf. ibid., 17).

3.6. L'exemple de la récente insurrection en Grèce, portée par toute une infrastructure contre-culturelle, avec un appui substantiel dans les universités et bénéficiant d'un degré remarquable de solidarité parmi la population d'Athènes, met en lumière que le moulage autoritaire de la société selon les impératifs des marchés financiers dérégulés à l'échelle européenne (et au-delà) est susceptible de se heurter à des résistances d'ampleur. Prenons les événements grecs comme memento mori du système capitaliste sous sa forme néolibérale, et nous voyons qu'il n'est pas impossible de briser le consensus fatal qui pèse sur la société comme un incubus. Faire apparaître le fonctionnement des rouages du système capitaliste dans toute son absurdité, et mettre en évidence la nécessité d'entrer dans une logique de rejet : cela ne relève plus de l'utopie, car ce topos s'est déjà incarné dans un mouvement insurrectionnel. Or, quand le rejet de l'existant inacceptable fait place à la construction organique d'un contre-modèle de société émancipée, portée par une masse critique de la population et du tissu institutionnel, c'est exactement ce que l'on peut appeler à juste titre un projet contre-hégémonique, naissant de l'alliance entre la classe ouvrière, première victime déjà marginalisée par les choix gouvernementaux, et l'intelligence ancrée dans les AIE de l'enseignement secondaire et supérieur. C'est cela la conséquence du «saut de tigre dans le passé » - proche. Sans forcer l'optimisme outre mesure, il n'est pas absurde de pronostiquer que la trajectoire de l'évolution de notre système de société a une chance suffisamment probable de rencontrer, à moyen terme, une bifurcation, un clinamen du moment décisif irréversible et inéluctable. Il est, certes, possible d'attendre patiemment, et passivement, le verdict du destin. Il semble néanmoins préférable d'avancer consciemment vers ce "point de non-retour ", voire de le forcer quelque peu par l'action - par exemple par l'écriture de textes qui pourront guider la pratique, en guise de boussole idéologique.

\section{BIBLIOGRAPHIE}

Adorno, Theodor W, Erziehung zur Mündigkeit, Francfort-sur-le-Main, Suhrkamp,1971, 147 pages.

Adorno, Theodor W., Etudes sur la personnalité autoritaire, Paris, Allia, 2007, 435 pages.

Althusser, Louis, «Idéologie et appareils idéologiques d'État. (Notes pour une recherche) », in Althusser, Louis, Positions (1964-1975), Paris, Les Éditions sociales, 1976, 67-125.

Auernheimer, Georg, « Erziehung », in Historisch-kritisches Wörterbuch des Marxismus (HKWM), vol. 3, Berlin, Argument, 1998, Deuxième édition, 831-839.

Badiou, Alain, De quoi Sarkozy est-il le nom ?, Paris, Éd. Lignes, 2007, 155 pages.

Barben, Daniel, « Funktionalismus », in Historisch-kritisches Wörterbuch des Marxismus (HKWM), vol. 4, Berlin, Argument, 1999, 1141-1157.

Barthes, Roland, Le grain de la voix. Entretiens 1962-1980, Paris, Éd. du Seuil, 1981, 394 pages. 
Benjamin Walter, « Über den Begriff der Geschichte », in Benjamin, Walter, Kairos. Schriften zur Philosophie, Francfort-sur-le-Main: Suhrkamp, 2007, 313-324.

Birkmann, Konrad, Michael Winkler, « Edukationismus », in Historisch-kritisches Wörterbuch des Marxismus (HKWM), vol. 3, Berlin, Argument, 1998, Deuxième édition, 12-19.

Bott, Gerhard, Erziehung zum Ungehorsam, Berlin, März-Verlag. 1970, Deuxième édition, 125 pages. Bourdieu, Pierre, Contre-feux 2, Paris, Éd. Raisons d'Agir, 2001, 108 pages.

Decocqman, Claudine, Robert Jourdan, Ursula Reynis, Susanne Trabaud, Projekt Deutsch Neu. Allemand Premières, Paris, Nathan, 2007, 223 pages.

Hardt, Michael, Antonio Negri, Multitude. War and Democracy in the Age of Empire, London, New York, Toronto, Penguin Books, 2004, 426 pages.

Hardt, Michael, Antonio Negri, Commonwealth, Cambridge (Mass.), The Belknap Press of Harvard University Press, 434 pages.

Haug, Frigga, "Disziplin.", in Historisch-kritisches Wörterbuch des Marxismus (HKWM), vol. 2, Berlin, Argument, 1999, Deuxième edition, 787-801.

Illich, Ivan, Une société sans école, Paris, Éd. du Seuil, 2003, 220 pages.

Lichtman, Richard, « Freudomarxismus », in Historisch-kritisches Wörterbuch des Marxismus (HKWM), vol. 4, Berlin, Argument, 1999, 1008-1026.

Maffesoli, Michel, « Éditorial : Être ensemble mais hors du lit de Procuste. La crise des élites », in Maffesoli, Michel; Christian Bourion (éds.), Ruptures et liens, Paris, Éd. ESKA, 2007, 9-21.

Marcuse, Herbert, Eros et civilisation, Paris, Éd. de Minuit, 1963, 239 pages.

Marcuse, Herbert, L'Homme unidimensionnel. Essai sur l'idéologie de la société industrielle avancée, Paris, Éd. de Minuit, 1968, 281 pages.

Marcuse, Herbert, A Study on Authority, London, New York, Verso, 2008a, 111 pages.

Marcuse, Herbert, Tolérance Répressive, suivi de Quelques conséquences sociales de la technologie moderne, Paris, Homnisphères, 2008b, 144 pages.

Mouffe, Chantal, On the Political. Thinking in Action, London, New York, Routledge, 2008, 144 pages.

Osterkamp, Ute, « Gefühle, Emotionen », in Historisch-kritisches Wörterbuch des Marxismus (HKWM), vol. 4, Berlin, Argument, 1999, 1329-1347.

Pêcheux, Michel, Les vérités de La Palice, Paris, Maspero, 1975, 279 pages.

Pêcheux, Michel, « Zu rebellieren und zu denken wagen! Ideologien, Widerstände, Klassenkampf », KultuRRevolution, vol. 3, n 5, 1984a, ISSN 0723-8088, 61-65.

Pêcheux, Michel, « Zu rebellieren und zu denken wagen! Ideologien, Widerstände, Klassenkampf », KultuRRevolution vol. 3, n 6, 1984b, ISSN 0723-8088, 63-66.

Reich, Wilhelm, La psychologie de masse du fascisme, Paris, Payot, 1974, 341 pages.

Sorel, Georges, Réflexions sur la violence, Paris, Éd. du Seuil, 1990, 324 pages.

Weiss, Ulrich, « Emanzipation », in Historisch-kritisches Wörterbuch des Marxismus (HKWM), vol. 3, Berlin, Argument, 1998, Deuxième édition, 272-289. 


\section{AUTEUR}

FRANK JABLONKA

Docteur en sciences du langage, Université de Picardie Jules Vernes, Amiens. 